ESAIM: PROCEEDINGS AND SURVEYS, 2020, Vol. 68, p. 52-72

Hervé Cardot \& Pierre Calka

\title{
DYNAMICS OF INTERACTING PARTICLE SYSTEMS ${ }^{*, * *, * * *}$
}

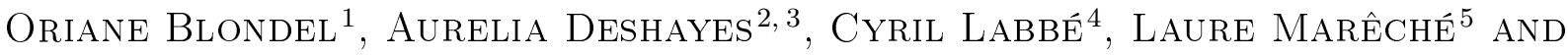 \\ MARIELLE SIMON ${ }^{6}$
}

\begin{abstract}
We collect here recent results covering various aspects of the dynamical properties of interacting particle systems. In Section 1 we study the hydrodynamic limit of a facilitated exclusion process. Section 2 evidences a cutoff phenomenon for the mixing time of the weakly asymmetric exclusion process. Section 3 presents a study of the infection time in the Duarte model. Finally, Section 4 presents the study of a front propagation in the FA-1f model.

Résumé. Nous rassemblons ici des résultats récents sur différentes propriétés dynamiques des systèmes de particules en interaction. En section 1 on étudie la limite hydrodynamique d'un processus d'exclusion facilité. La section 2 met en évidence un phénomène de cutoff pour le temps de mélange du processus d'exclusion faiblement asymétrique. La section 3 présente une étude du temps d'infection du modèle de Duarte. Enfin, la section 4 présente l'étude de la propagation d'un front dans le modèle FA-1f.
\end{abstract}

\section{INTRODUCTION}

Interacting particle systems appear naturally as microscopic models for various dynamical phenomena. Here we will consider a subfamily of these models consisting of continuous time Markov processes on a state space that can be represented as $\{0,1\}^{\Lambda}$, with $\Lambda=\mathbb{Z}^{d},\{1, \ldots, N\}^{d}$ or $\mathbb{T}_{N}=(\mathbb{Z} / N \mathbb{Z})^{d}$. Let us consider that 1 denotes the presence of a particle and 0 an empty site, though different interpretations are possible depending on the physical motivation for the model, as will be seen in the different sections. These motivations, far from being inconsequential, will lead the mathematician to focus on different aspects of the corresponding model. The dynamics considered will be either of Kawasaki type, i.e. conservative (an update corresponds to a particle

* O.B. acknowledges support from the ANR projects LSD (ANR-15-CE40-0020) and MALIN (ANR-16-CE93-0003).

** M.S. acknowledges support from the Labex CEMPI (ANR-11-LABX-0007-01), from the project EDNHS (ANR-14-CE250011) of the French National Research Agency (ANR) and from the European Research Council (ERC) under the European Union's Horizon 2020 research and innovative programme (grant agreement $n^{\circ}$ 715734).

*** L.M. acknowledges support from the ERC Starting Grant 680275 MALIG.

${ }^{1}$ Univ Lyon, CNRS, Université Claude Bernard Lyon 1, UMR5208, Institut Camille Jordan, F-69622 Villeurbanne, France. e-mail: blondel@math.univ-lyon1.fr

${ }^{2}$ Université Paris-Est, Laboratoire d'Analyse et de Mathématiques Appliquées (UMR 8050), UPEM, UPEC, CNRS, F-94010, Créteil, France

${ }^{3}$ Learning, Data \& Robotics Lab - ESIEA, Paris, France. e-mail: deshayes@esiea.fr

${ }^{4}$ Université Paris-Dauphine, PSL Research University, Ceremade, CNRS, 75775 Paris Cedex 16, France.

e-mail: labbe@ceremade.dauphine.fr

${ }^{5}$ LPSM UMR 8001, Université Paris Diderot, Sorbonne Paris Cité, CNRS, 75013 Paris, France. e-mail: mareche@lpsm.paris.

${ }^{6}$ Inria, Univ. Lille, CNRS, UMR 8524 - Laboratoire Paul Painlevé, F-59000 Lille, France. e-mail: marielle.simon@inria.fr

(C) EDP Sciences, SMAI 2020

This is an Open Access article distributed under the terms of the Creative Commons Attribution License (http://creativecommons.org/licenses/by/4.0), which permits unrestricted use, distribution, and reproduction in any medium, provided the original work is properly cited. 
jumping to an empty site), or of Glauber type, i.e. non-conservative (an update corresponds to a particle being deleted or created on an empty site).

In the first category, we will consider the very standard simple exclusion process (Section 2), as well as a facilitated version (Section 1), both in dimension 1. In these models, single particles occupy the sites of a lattice, and each one can jump at some rate to an empty neighboring vacant site. More precisely, to each lattice site is attached an independent random Poisson clock. When the clock rings, if there is a particle at this site, then it jumps to a neighboring site with a probability depending on the local configuration. In this class of models the total number of particles is always conserved along the evolution. This family of interacting particle processes has turned out to be rich enough to describe many physical systems and capture very complex features [3,20,26].

For these diffusive systems, maybe the most natural question is to understand how the density of particles evolves in time: choose the initial configuration in agreement with a certain (not necessarily constant) density profile; what density profile describes the configuration at a later time? This is the problem of the hydrodynamic limit. In general we expect that in the right time scale (given by that of the underlying random walks), the answer will be given by the solution to a certain PDE depending on the model. For the simple exclusion process, this has been known for decades [13,19,20]: the PDE is the heat equation in the symmetric case, the inviscid Burgers equation in the asymmetric case and a viscous Burgers equation in certain weakly asymmetric cases. The interest of the (symmetric) facilitated exclusion process considered in Section 1 stems from the fact that its microscopic mechanism should induce a separation between two phases in the limit, thus modelling melting phenomena. This is a consequence of choosing degenerate jump rates, meaning that the probability for a particle to jump to an empty neighbor vanishes as soon as some local constraint is not satisfied. In Section 1 we present a result describing the hydrodynamic limit of the liquid phase.

The hydrodynamic limit gives a first idea of the long-time distribution of a conservative system. A related but different question is to quantify the distance to equilibrium of the system in a (large) finite domain. In many systems we expect that the total variation distance undergoes a sharp transition: that is the cutoff phenomenon. It has been established in a variety of models, and an ongoing research effort consists in identifying necessary and/or sufficient conditions for that phenomenon to occur. Section 2 presents new cutoff results for the weakly asymmetric exclusion process, in which the particles have a bias which vanishes with the size of the domain.

For non-conservative systems, the relaxation to equilibrium follows a very different pattern, since it is not governed by random walk diffusion. The models that we will consider in this category are Kinetically Constrained Models (KCM), which have been introduced as toy models for supercooled liquids approaching the liquid-glass transition [11,32], a major open problem in condensed matter physics. A configuration of a $\mathrm{KCM}$ is given by assigning to each vertex $x \in \mathbb{Z}^{d}$ an occupation variable which corresponds to an empty or occupied site (in Section 3, we will rather use the terminology infected/healthy to make the comparison with bootstrap percolation more transparent). The evolution is given by a Markovian stochastic dynamics of Glauber type. With rate one, each vertex updates its occupation variable to occupied or to empty with probability $p \in[0,1]$ and $q=1-p$, respectively, if the configuration satisfies a certain local constraint. The constraint is chosen in a way that does not depend on the updated vertex, so that the product Bernoulli measure $\mu_{q}:=\operatorname{Ber}(1-q)^{\otimes \mathbb{Z}^{d}}$ is reversible w.r.t. such processes.

Similarly to the facilitated exclusion process of Section 1, the presence of degenerate rates in the dynamics leads to the existence of frozen structures and it is interesting to understand how these affect the mixing properties of the dynamics. It is known [11] that inside the ergodic phase (with no infinite frozen structure), the relaxation time to equilibrium does not diverge with the size of the domain. A physically relevant question is to understand how this relaxation time or other related quantities diverge as the density is increased toward the ergodicity threshold. Section 3 analyzes this divergence for the infection time in the Duarte model, a twodimensional KCM, and compares it with the divergence of the analogous quantity in the corresponding bootstrap percolation model.

Out-of-equilibrium dynamics of KCM, i.e. when the process is started from a measure different from $\mu_{q}$, are much less understood. In Section 4, we see that in the one-dimensional FA-1f model, a vacancy propagates 
equilibrium linearly in an initially frozen region. This result belongs to the family of shape theorems for models with propagation of infection.

\title{
1. FAcilitated EXClusion PROCESSES
}

\author{
Marielle Simon
}

This is based on a recent collaborative work [6] with O. Blondel, C. Erignoux and M. Sasada.

\subsection{Multiphase media and kinetically constrained lattice gases}

\subsubsection{PDEs for multiphase media}

We are interested here in the models that describe the evolution of a multiphase medium (for instance the joint evolution of liquid and solid phases). These physical phenomena typically involve non-equilibrium phase transitions which can be found in nature, ranging from morphological transitions of growing surfaces to moving fronts of populations, or traffic jams. The porous medium equation written as

$$
\partial_{t} \rho=\operatorname{div}\left(\rho^{m-1} \nabla \rho\right)
$$

is one of the simplest examples of nonlinear evolution equation of parabolic type. The constant $m>1$ regulates the behavior of the diffusion coefficient $\left(D(\rho)=\rho^{m-1}\right)$ close to vanishing densities. Above, div and $\nabla$ are respectively the divergence and gradient operators in $\mathbb{R}^{d}$. The evolution equation (1) is often used to describe the evolution of the scaled density $\rho: \mathbb{R}^{d} \times \mathbb{R}_{+} \rightarrow[0,1]$ of an ideal gas flowing in a homogeneous medium. It is known that, starting from an initial density $\rho_{0}$, the solution $\rho(x, t)$ is nonnegative and has compact support in the space variables for each positive $t$. Thus there are interfaces separating the regions where $\rho$ is positive from those where it is zero. When $m=1$ (resp. $m<1$ ), equation (1) is called heat equation (resp. fast diffusion equation).

More generally, the Stefan problem introduced in [35] typically describes the temperature distribution in a homogeneous medium which is subjected to a phase change between ice and water. Let us assume first that heat diffuses linearly, and let us write the evolution equation of both separate media, with the separation interface allowed to evolve in time. We define the water region as the domain where $\rho>0$ and the ice region where $\rho=0$. In one dimension, the mathematical formulation is the following: find a curve $x=\Gamma(t)$ and a function $\rho(x, t) \geq 0$ such that

$$
\left\{\begin{array}{l}
\partial_{t} \rho=D \partial_{x x}^{2} \rho, \quad \text { if } 0<x<\Gamma(t), \\
\rho(x, t)=0,
\end{array} \quad \text { if } x \geq \Gamma(t), \quad \text { and } \quad \frac{d \Gamma}{d t}=-\partial_{x} \rho(\Gamma(t), t)\right.
$$

where $D>0$ is a diffusion constant, plus initial and boundary conditions. The second condition on the free boundary $\Gamma(t)$ physically corresponds to the presence of latent heat at the phase transition. The mathematical solution to (2) is usually obtained via the weak formulation and the regularity of $\Gamma(t)$ can be analyzed (see [30] for a recent review on the Stefan problem). The combination of nonlinear diffusion (similar to (1)) with the Stefan problem has been less studied and that is what we will focus on.

\subsubsection{Facilitated exclusion process}

Some macroscopic equations featuring free boundaries have been recently derived from microscopic particle systems, for instance in $[14,16]$, however in non-degenerate settings. Our ultimate goal (and prospective work) is to derive the Stefan problem from a conservative and degenerate microscopic dynamics. This is why we chose to work with kinetically constrained lattice gases which are usually studied in the context of glassy dynamics (as examples of systems in which the transition out of a liquid state is due to dynamical arrest), see [1,17] or [32] for a review. 


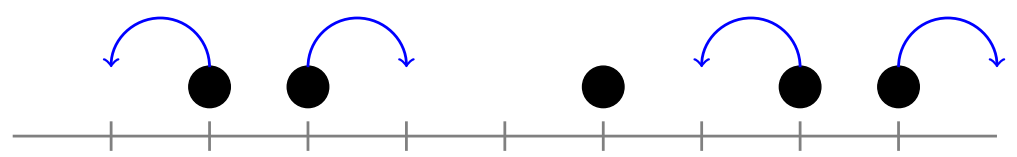

FiguRE 1. Constrained jumps of particles: the only possible jumps are represented by directed arrows.

In $[6,33]$ a microscopic dynamics called facilitated exclusion process is investigated (see also [1,17]). Particles are distributed on the points of the finite torus of size $N$, with the exclusion restriction, meaning that no two particles can occupy the same site. A particle at $x$ jumps to an empty neighboring site, say $x+1$, at rate 1 if there is a particle at $x-1$, and rate 0 else. The jump rate from $x+1$ to $x$ is given by a similar rule: a particle at $x+1$ can jump to site $x$ at rate 1 if there is a particle at $x+2$, and rate 0 else. Examples of jumps are given in Figure 1. Because of the extra constraint to allow a jump, the dynamics is referred to as degenerate.

This dynamics conserves the total number of particles $\sum_{x} \eta(x)$ and is reversible. However, its invariant measures are not product, contrary to the vast majority of similar models (see Proposition 1.3). Because of the jump constraint, we identify two distinct regimes for the macroscopic behavior of this model. Either the macroscopic density is larger than $\frac{1}{2}$, in which case the system behaves diffusively, or the density is lower than $\frac{1}{2}$, in which case the system remains frozen. The interfaces between these two phases propagate as particles from the supercritical phase $\left(\rho>\frac{1}{2}\right)$ diffuse towards the subcritical phase $\left(\rho<\frac{1}{2}\right)$. We expect that the macroscopic density profile for this exclusion dynamics evolves under the diffusive time scaling according to the Stefan problem

$$
\partial_{t} \rho=\partial_{x x}(G(\rho)) \quad \text { where } G(\rho)=\frac{2 \rho-1}{\rho} \mathbf{1}_{\left\{\rho>\frac{1}{2}\right\}} .
$$

In [6] we are able to treat the liquid part of the problem and we provide an estimation of the time needed by the system to enter into the ergodic state: if the density is initially larger than $\frac{1}{2}$ and if the microscopic system is of size $N$, then at a macroscopic time of order $(\log N)^{\alpha} / N^{2}$, with high probability the system of particles has already reached the ergodic component. This is the content of Theorem 1.6 below.

Let us give an outline of what follows: in Section 1.2 we give a precise definition of the interacting particle system, and investigate in detail its characteristics. In Section 1.3 we state the main results of [6], which concern the sub-diffusive estimate of the transience time, and the hydrodynamic limits in the subcritical phase $\left(\rho>\frac{1}{2}\right)$.

\subsection{Microscopic characteristics}

Let $\mathbb{T}_{N}:=\mathbb{Z} / N \mathbb{Z}$ be the finite one-dimensional torus of size $N$. The facilitated exclusion process $\left\{\eta_{t}(x) ; x \in\right.$ $\left.\mathbb{T}_{N}\right\}$ is a Markov process on $\{0,1\}^{\mathbb{T}_{N}}$, whose time evolution is ruled by the infinitesimal generator $\mathcal{L}_{N}$, which acts on functions $f:\{0,1\}^{\mathbb{T}_{N}} \rightarrow \mathbb{R}$ as

$$
\mathcal{L}_{N} f(\eta):=\sum_{x \in \mathbb{T}_{N}} c_{x, x+1}(\eta)\left(f\left(\eta^{x, x+1}\right)-f(\eta)\right)
$$

where the constraint and the exclusion rule are encoded in the rates $c_{x, x+1}$ as

$$
c_{x, x+1}(\eta)=\eta(x-1) \eta(x)(1-\eta(x+1))+\eta(x+2) \eta(x+1)(1-\eta(x)),
$$

and $\eta^{x, y}$ denotes the configuration obtained from $\eta$ by exchanging the states of sites $x, y$, namely $\eta^{x, y}(x)=\eta(y)$, $\eta^{x, y}(y)=\eta(x)$ and $\eta^{x, y}(z)=\eta(z)$ if $z \neq x, y$. Figure 1 shows examples of jumps. The dynamics obviously conserves the total number of particles $\sum_{x \in \mathbb{T}_{N}} \eta(x)$. Because the jump rates can vanish, the dynamics is referred to as degenerate.

We start by giving the classification of the configurations into transient/recurrent states. For any $k \in$ $\{1, \ldots, N\}$, we denote by $\mathcal{H}_{N}^{k}$ the hyperplane which contains all configurations $\eta$ with exactly $k$ particles, 


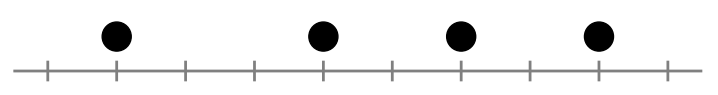

Figure 2. One absorbing (blocked) configuration, with $N=10$ and $k=4$

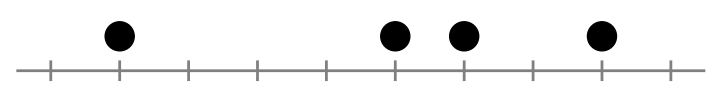

Figure 3. One transient bad configuration, with $N=10$ and $k=4$

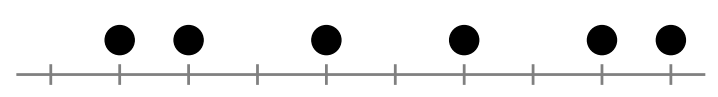

Figure 4. One ergodic configuration, with $N=10$ and $k=6$

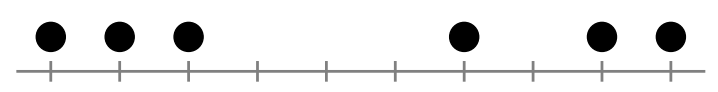

FiguRE 5. One transient good configuration, with $N=10$ and $k=6$

namely:

$$
\mathcal{H}_{N}^{k}:=\left\{\eta \in\{0,1\}^{\mathbb{T}_{N}}: \sum_{x \in \mathbb{T}_{N}} \eta(x)=k\right\}
$$

One can easily check the following statements:

(1) if $k \leq \frac{N}{2}$, then a configuration $\eta \in \mathcal{H}_{N}^{k}$ satisfies one of these two properties:

- either it is completely blocked, in the sense that no particle can perform a jump (since the constraint (5) is never satisfied). These configurations are absorbing states for the dynamics: they are exactly the configurations in which all particles are isolated, see Figure 2;

- or there exist in $\eta$ some particles which are able to jump. However, starting from one of these configurations, with probability one, in a finite number of steps the process will end in a blocked configuration. These configurations are called transient bad, see Figure 3.

(2) if $k>\frac{N}{2}$, similarly a configuration $\eta \in \mathcal{H}_{N}^{k}$ satisfies one of these two properties:

- either $\eta$ is in the ergodic component, i.e. the set of recurrent states for the process, which here form an irreducible component; we call them ergodic configurations. They are the configurations in which empty sites are isolated, see Figure 4;

- or, starting from $\eta$, the process enters the ergodic component after a finite number of steps a.s. These configurations are called transient good configurations, see Figure 5.

Those observations lead to the following definition.

Definition 1.1. We denote by $\mathcal{E}_{N} \subset\{0,1\}^{\mathbb{T}_{N}}$ the set of ergodic configurations on $\mathbb{T}_{N}$, namely

$$
\mathcal{E}_{N}:=\left\{\eta \in\{0,1\}^{\mathbb{T}_{N}}: \forall x \in \mathbb{T}_{N},(\eta(x), \eta(x+1)) \neq(0,0) \text { and } \sum_{x \in \mathbb{T}_{N}} \eta(x)>\frac{N}{2}\right\} .
$$

For $k>\frac{N}{2}$ we define

$$
\Omega_{N}^{k}:=\mathcal{H}_{N}^{k} \cap \mathcal{E}_{N}
$$

as the set of ergodic configurations on $\mathbb{T}_{N}$ which contain exactly $k$ particles.

We are now able to give the following two results on the invariant measures, taken directly from [6]: 
Lemma 1.2. For any $N \geq 1$ and $k>\frac{N}{2}, \Omega_{N}^{k}$ is an irreducible component for the facilitated exclusion process.

Proof. Let us change the point of view and move the zeros around instead of the particles. Then it is clear that in a configuration in $\mathcal{E}_{N}$, a zero can jump as long as it remains at distance at least two from the others, and every allowed jump is reversible. Consequently, it is enough to show that from every configuration in $\Omega_{N}^{k}$, one can reach the configuration $\circ \bullet \circ \bullet \cdots \bullet \bullet$ where the $N-k$ zeros start alternating with particles until there are none left. Let $\eta \in \Omega_{N}^{k}$ and number its zeros from left to right (the "left-most" site being $1 \in \mathbb{T}_{N}$ ). In that order, pull each of them as much to the left as possible. That way we reach either the desired configuration, or the same shifted one step to the right (in case $\eta(N)=0$ ): $\bullet \circ \bullet \bullet \cdots \bullet$. In the second case, iterating the process brings us back to the desired configuration.

Proposition 1.3. (1) For any $k>\frac{N}{2}$, the (canonical) uniform measures on $\Omega_{N}^{k}$, denoted by $\pi_{N}^{k}$, are invariant for the Markov process induced by the infinitesimal generator $\mathcal{L}_{N}$ and satisfy the detailed balance condition: for any $x \in \mathbb{T}_{N}$ and $\eta \in \Omega_{N}^{k}$,

$$
\pi_{N}^{k}(\eta) \eta(x-1) \eta(x)(1-\eta(x+1))=\pi_{N}^{k}\left(\eta^{x, x+1}\right)\left(1-\eta^{x, x+1}(x)\right) \eta^{x, x+1}(x+1) \eta(x+2) .
$$

(2) When $k / N \rightarrow \rho>\frac{1}{2}$, the sequence $\left\{\pi_{k}^{N}\right\}$ locally converges to a grand-canonical measure $\pi_{\rho}$ on $\{0,1\}^{\mathbb{Z}}$.

This measure satisfies: for any $\eta \in\{0,1\}^{\mathbb{Z}}$,

(a) $\pi_{\rho}(\eta(0))=\rho$.

(b) for any $\ell \in \mathbb{N}, \ell \geq 1$

$$
\pi_{\rho}\left(\eta_{\mid\{1, \ldots, \ell\}}=(0,1, \ldots, 1)\right)=(1-\rho)\left(\frac{2 \rho-1}{\rho}\right)^{\ell-2}
$$

which, since $\frac{1-\rho}{\rho}=1-\frac{2 \rho-1}{\rho}$, is nothing but

$$
\pi_{\rho}(\eta(0)=0) \times \mathbb{P}\left(\operatorname{Geom}\left(\frac{1-\rho}{\rho}\right) \geq \ell-2\right) .
$$

Finally, let us say a few words about the instantaneous currents, which are defined for any configuration $\eta$ and any site $x$ as

$$
j_{x, x+1}(\eta)=c_{x, x+1}(\eta)(\eta(x+1)-\eta(x)),
$$

and satisfy $\mathcal{L}_{N}(\eta(x))=j_{x, x+1}(\eta)-j_{x-1, x}(\eta)$. One can easily check that our model is gradient, which is to say that these currents can be written as discrete gradients: for any $x \in \mathbb{T}_{N}$,

$$
j_{x, x+1}(\eta)=\tau_{x} h(\eta)-\tau_{x+1} h(\eta)
$$

with the function $h$ given by $h(\eta):=\eta(-1) \eta(0)+\eta(0) \eta(1)-\eta(-1) \eta(0) \eta(1)$. As in all gradient models, this function $h$ plays a fundamental role in the derivation of the hydrodynamic limit.

\subsection{Transience time and hydrodynamic limits}

We are now ready to state the main results of [6]. In order to derive the macroscopic limit of the facilitated exclusion process, we first fix an initial smooth profile $\rho_{0}: \mathbb{T} \rightarrow\left(\frac{1}{2}, 1\right]$, where $\mathbb{T}=\mathbb{R} / \mathbb{Z}$ is the continuous onedimensional torus. We let the Markov process start from the non-homogeneous product measure on $\{0,1\}^{\mathbb{T}_{N}}$ fitting $\rho_{0}$, defined as

$$
\mu_{N}(\eta):=\prod_{k \in \mathbb{T}_{N}}\left(\rho_{0}\left(\frac{k}{N}\right) \eta(k)+\left(1-\rho_{0}\left(\frac{k}{N}\right)\right)(1-\eta(k))\right) .
$$

Let $\left\{\eta_{t}^{N}: t \geq 0\right\}$ denote the Markov process driven by the accelerated infinitesimal generator $N^{2} \mathcal{L}_{N}$ starting from the initial measure $\mu_{N}$. Fix $T>0$ and denote by $\mathbb{P}_{\mu_{N}}$ the probability measure on the Skorokhod path space $\mathcal{D}\left([0, T],\{0,1\}^{\mathbb{T}_{N}}\right)$ corresponding to this dynamics. 
Theorem 1.4 (Hydrodynamic limit, [6]). For any $t \in[0, T]$, any $\delta>0$ and any continuous test function $\varphi: \mathbb{T} \rightarrow \mathbb{R}$, we have

$$
\lim _{N \rightarrow \infty} \mathbb{P}_{\mu_{N}}\left[\left|\frac{1}{N} \sum_{x \in \mathbb{T}_{N}} \varphi\left(\frac{x}{N}\right) \eta_{t}^{N}(x)-\int_{\mathbb{T}} \varphi(u) \rho(t, u) d u\right|>\delta\right]=0
$$

where $\rho(t, u)$ is the unique smooth solution of the hydrodynamic equation

$$
\partial_{t} \rho=\partial_{x x}\left(\frac{2 \rho-1}{\rho}\right), \quad \rho(0, \cdot)=\rho_{0}(\cdot): \mathbb{T} \rightarrow\left(\frac{1}{2}, 1\right] .
$$

Remark 1.5. The equation (11) belongs to the family of quasilinear parabolic problems, for which it is quite standard to prove that there is a unique smooth solution, provided that the initial profile satisfies $\rho_{0}>\frac{1}{2}$.

There are two main difficulties to prove Theorem 1.4. The first one lies in the fact that the dynamics is a priori not ergodic. We therefore need a second result, which states that the accelerated system reaches its ergodic component at a macroscopic time $t_{N}$ of order $o(1)$ as $N \rightarrow \infty$. Therefore, for any macroscopic time $t>0$ and for any $N$ large enough such that $t_{N}<t$, the configuration $\eta_{t}^{N}$ belongs to the ergodic component with very high probability. This is the purpose of:

Theorem 1.6 (Transience time for the exclusion process with absorption, [6]). Letting $\ell_{N}=(\log N)^{32}$ and $t_{N}=\ell_{N} / N^{2}$, we have

$$
\lim _{N \rightarrow \infty} \mathbb{P}_{\mu_{N}}\left(\eta_{t_{N}}^{N} \notin \mathcal{E}_{N}\right)=0
$$

The main idea of its proof is to use an already known mapping, which couples the process $\left\{\eta_{t}^{N}: t \geq 0\right\}$ generated by $\mathcal{L}_{N}$ with a zero-range process which presents the same characteristic separation between ergodic, transient and inactive states, but has additional pleasant monotonicity properties. These make the estimation of the time needed to enter into the ergodic component less complicated, although still technical. All the details are contained in [6, Section 4].

The second main difficulty to prove Theorem 1.4 comes from the nature of the invariant measures of the process: we need a deep investigation of the canonical and grand canonical measures for the generator $\mathcal{L}_{N}$, which only charge the ergodic component $\mathcal{E}_{N}$ where all empty sites are isolated. There are two fundamental properties of $\pi_{\rho}$ which we prove in [6]:

(1) For any density $\rho \in\left(\frac{1}{2}, 1\right)$, under $\pi_{\rho}$, the correlations between two boxes at a distance of order $\ell$ decay as $\exp (-C \ell)$. This is the purpose of [6, Lemma 6.5].

(2) The equivalence of ensembles, linking canonical measures to grand-canonical measures as the system size goes to infinity, holds. This is the content of [6, Corollary 6.10].

Then, our proof of the hydrodynamic limit relies on the classical entropy method (which is explained in details for instance in [20, Chapter 5]), but requires adaptations to solve the ergodicity issue, and to account for the non-product invariant measures. The main steps of the proof are given in [6, Section 5] in the form of two distinct results:

- the first one states that after reaching the ergodic component, the macroscopic density profile of the system is very close to the initial density profile $\rho_{0}$. This is [6, Lemma 5.1];

- the second result states that starting from the ergodic component, the hydrodynamic limit holds. This is [6, Proposition 5.2]. Its proof uses the classical Replacement Lemma, which, because of the shape of our canonical and grand canonical measures, requires significant work. In particular, this is where the two fundamental properties of $\pi_{\rho}$ - the correlations decay and the equivalence of ensembles - are crucial. 


\title{
2. Cutoff Phenomenon For the WEAKLy ASYMMETRIC SIMPle EXCLUSION PROCESS
}

\author{
Cyril Labbé
}

\subsection{Introduction}

The simple exclusion process is a model of statistical mechanics that provides a simplified picture for a gas of interacting particles. Particles move on a lattice, each of them performing a nearest neighbor random walk independently of the others, and interact only via the exclusion rule that prevents any two particles from sharing the same site (when a particle tries to jump on a site which is already occupied, this jump is cancelled).

While convergence to equilibrium for a particle system can be considered on a macroscopic scale via the evolution of the particle density or hydrodynamic profile (see e.g. Section 1, [20] and references therein), an alternative and complementary viewpoint consists in measuring the so-called $\varepsilon$-Total Variation Mixing Time [25]. It is defined as the first time at which the total variation distance to the stationary state, starting from the "worst" initial condition, falls below a given threshold $\epsilon$. Compared to the hydrodynamic profile, this provides a much more microscopic information on the particle system.

We will review the recent progress made on the derivation of the asymptotics of the mixing times for the simple exclusion process on a segment.

\subsection{The Markov chain and its equilibrium measure}

Given $N \in \mathbb{N}, k \in \llbracket 1, N-1 \rrbracket$ (we use the notation $\llbracket a, b \rrbracket=[a, b] \cap \mathbb{Z}$ ) and $p \in(1 / 2,1]$, the Asymmetric Simple Exclusion Process on $\llbracket 1, N \rrbracket$ with $k$ particles and parameter $p$ is the random process on the state space

$$
\mathcal{H}_{N}^{k}:=\left\{\eta \in\{0,1\}^{N}: \sum_{x=1}^{N} \eta(x)=k\right\}
$$

associated with the generator

$$
\mathcal{L}_{N, k} f(\eta):=\sum_{y=1}^{N-1}\left(q \mathbf{1}_{\{\eta(y)<\eta(y+1)\}}+p \mathbf{1}_{\{\eta(y)>\eta(y+1)\}}\right)\left(f\left(\eta^{y, y+1}\right)-f(\eta)\right),
$$

where $q=1-p$ and $\eta^{y, y+1}$ is obtained from $\eta$ by exchanging its values at $y$ and $y+1$.

In a more intuitive manner we can materialize the positions of 1 by particles, and say that the particles perform random walks with jump rates $p$ to the right and $q=1-p$ to the left: these random walks are independent from one another except that any jump that would send a particle to a location already occupied by another particle is cancelled.

It is convenient to use another, equivalent, representation of a particle configuration through a so-called height function. With any configuration $\eta \in \mathcal{H}_{N}^{k}$, we associate a lattice path $h=h_{\eta}$ that makes \pm 1 steps according to the presence/absence of particles at the corresponding sites:

$$
h(0)=0, \quad h(x)-h(x-1)=2 \eta(x)-1, \quad x \in \llbracket 1, N \rrbracket .
$$

We say that $h$ has an upward corner (resp. downward corner) at $x$ if $h(x+1)=h(x-1)=h(x)-1($ resp. $h(x+1)=h(x-1)=h(x)+1)$. Note that an upward corner at $x$ corresponds to having a particle at $x$ and no particle at $x+1$; similarly a downward corner at $x$ corresponds to having no particle at $x$ and a particle at $x+1$. Consequently, moving a particle from $x$ to $x+1$ corresponds to flipping an upward corner into a 
downward corner, and similarly, moving a particle from $x+1$ to $x$ corresponds to flipping a downward corner into an upward corner. One can therefore check that the dynamics of the particles can be rephrased at the level of the height functions in the following way: any upward corner (resp. downward corner) of $h$ flips into its opposite at rate $p$ (resp. $q$ ).

We let $P_{t}^{N, k}$ denote the associated semi-group. This Markov chain is irreducible, and admits a unique invariant (and reversible) probability measure $\pi_{N, k}$ given by

$$
\pi_{N, k}(\eta):=\frac{1}{Z_{N, k}}\left(\frac{q}{p}\right)^{\frac{1}{2} A(\eta)} .
$$

where $Z_{N, k}:=\sum_{\eta \in \mathcal{H}_{N}^{k}}\left(\frac{q}{p}\right)^{\frac{1}{2} A(\eta)}$, and $A(\eta)=\sum_{x=1}^{N} h_{\eta}(x)$ is the area below the height function $h_{\eta}$.

In the symmetric case $p=q=1 / 2$, this is simply the uniform measure on the set $\mathcal{H}_{N}^{k}$ while in the asymmetric case $p>q$, the measure $\pi_{N, k}$ favors configurations with a small area, or equivalently, whose height function is close to the minimal height function.

Recall that the total variation distance between two probability measures defined on the same state space $\Omega$ is defined by

where the sup is taken over all measurable sets $A$.

$$
\|\alpha-\beta\|_{T V}=\sup _{A \subset \Omega}\{\alpha(A)-\beta(A)\}
$$

The mixing time associated with the threshold $\varepsilon \in(0,1)$ is defined by

$$
T_{\text {mix }}^{N, k}(\varepsilon):=\inf \left\{t \geq 0: d^{N, k}(t) \leq \varepsilon\right\},
$$

where $d^{N, k}(t)$ denotes the total variation distance to equilibrium at time $t$ starting from the worst possible initial condition

$$
d^{N, k}(t):=\max _{\eta \in \mathcal{H}_{N}^{k}}\left\|P_{t}^{N, k}(\eta, \cdot)-\pi_{N, k}\right\|_{T V}
$$

From now on, and for the sake of simplicity, we restrict ourselves to the case of a non-trivial density of particles, that is $k / N \rightarrow \alpha \in(0,1)$.

\subsection{Results on the mixing times}

The first main contribution on this question is due to Wilson [36]. Therein he showed that in the symmetric case $p=q=1 / 2$, for any $\varepsilon \in(0,1)$, we have for all $N$ large enough

$$
\frac{1+o(1)}{\pi^{2}} N^{2} \log N \leq T_{\mathrm{mix}}^{N, k}(\varepsilon) \leq \frac{2+o(1)}{\pi^{2}} N^{2} \log N .
$$

Such a behavior of the mixing times is usually referred to as a pre-cutoff phenomenon: all the mixing times are comprised in between two terms of the same order but whose prefactors differ. It is generally believed that for non-pathological Markov chains, this implies a cutoff phenomenon: all the mixing times are equivalent to each other

$$
T_{\text {mix }}^{N, k}(\varepsilon) \sim T_{\text {mix }}^{N, k}(1 / 2), \quad \forall \varepsilon \in(0,1) .
$$

The second main contribution is the work of Benjamini et al. [2] where the asymmetric case ( $p$ is larger than $1 / 2$ and independent of $N$ ) is considered. It is shown therein that a precutoff phenomenon holds: there exist $0<C_{1}<C_{2}<\infty$ such that for any $\varepsilon \in(0,1)$, we have for all $N$ large enough

$$
C_{1} N \leq T_{\text {mix }}^{N, k}(\varepsilon) \leq C_{2} N
$$




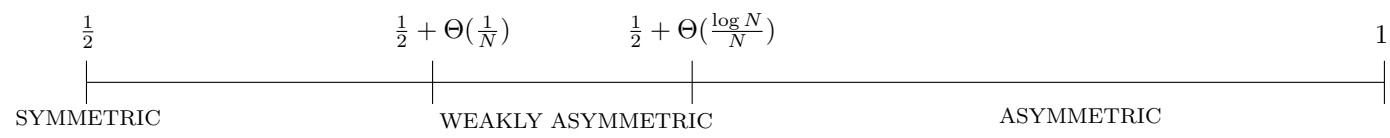

Wilson '04

$\asymp N^{2} \log N$

Benjamini et al. '05

$$
\asymp N
$$

$\underset{N^{2} \log N}{\operatorname{Lacoin}}$

$\sim \frac{N^{2} \log N}{\pi^{2}}$

Levin and Peres '16

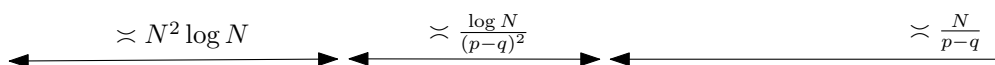

L. and Lacoin '16,'18

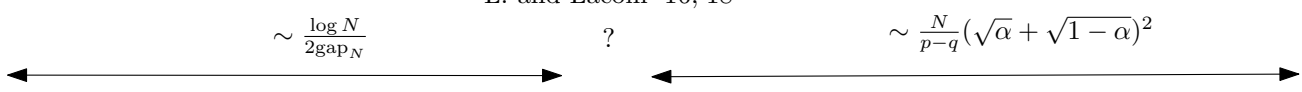

FIGURE 6. The main results on the mixing times of the SEP. $p$ increases from $1 / 2$ to 1 (not on scale) when reading the picture from left to right. We use the notation $\asymp u_{N}$ as a shorthand for the existence of two constants $C_{1}<C_{2}$ such that for every given $\varepsilon \in(0,1)$ we have $C_{1} u_{N} \leq T_{\text {mix }}^{N, k}(\varepsilon) \leq C_{2} u_{N}$ as soon as $N$ is large enough. Furthermore, we write $\sim u_{N}$ as a shorthand for $T_{\text {mix }}^{N, k}(\varepsilon) \sim u_{N}$ for any given $\varepsilon \in(0,1)$ as $N \rightarrow \infty$. $\Theta\left(u_{N}\right)$ denotes a quantity that is $\asymp u_{N}$.

Later on, Lacoin [23] showed that cutoff holds in the symmetric case $p=q=1 / 2$, namely for all $\varepsilon \in(0,1)$

$$
\lim _{N \rightarrow \infty} \frac{T_{\operatorname{mix}}^{N, k}(\varepsilon)}{N^{2} \log N}=\frac{1}{\pi^{2}} .
$$

Note that the lower bound of Wilson was sharp, but the upper bound was not.

The case of weak asymmetry (when $p=p_{N}$ and $q=q_{N}$ depend on $N$ with $b_{N}=p_{N}-q_{N}=o(1)$ ) was studied only recently by Levin and Peres [24]. Three regimes were identified in terms of the order of magnitude of the bias $b_{N}$ :

(A) When $b_{N} \leq 1 / N$, the mixing time remains of the same order as that of the symmetric case $N^{2} \log N$.

(B) When $1 / N \leq b_{N} \leq(\log N) / N$, the mixing time is of order $\left(b_{N}\right)^{-2} \log N$.

(C) When $(\log N) / N \leq b_{N} \leq 1$, the mixing time is of order $\left(b_{N}\right)^{-1} N$.

In all regimes, the upper and lower constants differ so that only precutoff phenomena are established. The transition occurring around $b_{N} \approx N^{-1}$ is the one observed for the hydrodynamic limit: it corresponds to a crossover regime where the limit is given by a viscous Burgers' equation $[13,19,20]$ which interpolates between the heat and the inviscid Burgers' equations. The one occurring for $b_{N} \approx N^{-1} \log N$ is however not observed in the macroscopic profile. It corresponds to a crossover regime for the position of the leftmost particle at equilibrium: for asymmetries much smaller than $N^{-1} \log N$, the leftmost particle is at distance negligible compared to $N$ of site 1, while for much larger asymmetries, it is at distance negligible compared to $N$ from site $N-k+1$ (note that the leftmost particle cannot be located to the right of this site).

In collaboration with Hubert Lacoin [21,22], we established a cutoff phenomenon for all regimes of (weak) asymmetry except when the bias is of order $(\log N) / N$. Actually, we identified two main regimes for the pattern of relaxation to equilibrium. The large bias regime where

$$
\lim _{N \rightarrow \infty} \frac{N b_{N}}{\log N}=\infty
$$


and the small bias regime where

$$
\lim _{N \rightarrow \infty} \frac{N b_{N}}{\log N}=0 .
$$

We identified the asymptotic expression for the mixing times in both regimes. In the large bias regime we showed that the mixing time coincides with the time needed by the particle density to reach equilibrium.

Theorem 2.1. When (17) holds and $\lim _{N \rightarrow \infty} k_{N} / N=\alpha \in(0,1)$, we have for every $\varepsilon \in(0,1)$

$$
\lim _{N \rightarrow \infty} \frac{b_{N} T_{\operatorname{mix}}^{N, k_{N}}(\varepsilon)}{N}=(\sqrt{\alpha}+\sqrt{1-\alpha})^{2} .
$$

To state our result in the small bias regime, let us introduce the quantity

$$
\operatorname{gap}_{N}:=\left(\sqrt{p_{N}}-\sqrt{q_{N}}\right)^{2}+4 \sqrt{p_{N} q_{N}} \sin \left(\frac{\pi}{2 N}\right)^{2},
$$

which corresponds to the spectral gap associated with the generator (12). Notice that it does not depend on the number $k$ of particles in the system. The pattern of relaxation is similar to the one observed in the symmetric case.

Theorem 2.2. When (18) holds, we have

$$
\lim _{N \rightarrow \infty} \frac{\operatorname{gap}_{N} T_{\operatorname{mix}}^{N, k_{N}}(\varepsilon)}{\log N}=\frac{1}{2}
$$

Using Taylor expansion for gap ${ }_{N}$ we have, whenever $b_{N}$ tends to zero

$$
\operatorname{gap}_{N} \stackrel{N \rightarrow \infty}{\sim} \frac{1}{2}\left(b_{N}^{2}+\left(\frac{\pi}{N}\right)^{2}\right) .
$$

These two theorems establish a cutoff phenomenon whenever the bias $b_{N}$ is much larger or much smaller than $(\log N) / N$. When the bias is of order $(\log N) / N$, the situation is more intricate and establishing cutoff remains an open question; however, the result of Levin and Peres already shows that a precutoff phenomenon holds in that case.

\section{BOOTSTRAP PERCOLATION VERSUS KINETICALLY CONSTRAINED MODEL: THE CASE OF THE DUARTE MODEL}

Laure Marêché

Bootstrap percolation and kinetically constrained models are interacting particle systems that are very similar: both kinds of models are dynamics of configurations on graphs where any vertex of the graph (we call them sites) can have two states, either infected or healthy, and the state of a site can change only if a constraint is satisfied at this site, the constraint being of the form "there are enough infected sites in a neighborhood of the site". In bootstrap percolation, a satisfied constraint will make the corresponding site infected, while in kinetically constrained models, it can make it infected or healthy at random. These models may be reminiscent of voter models, in which a site randomly takes the state of one of its neighbors, but they are fundamentally different, as healthy neighbors will not allow a site to become healthy. Despite their similarities, bootstrap percolation and kinetically constrained models have differences: the former has a deterministic dynamics, the latter have a stochastic one, the former is monotonic, the latter are not. Here we consider a specific, asymmetric constraint, the Duarte constraint, and we study the effects of these differences on the behaviors of the two models. We will see that though these two Duarte models share some properties, an important timescale, the first time at which the origin is infected, diverges much faster in the kinetically constrained model than in bootstrap percolation when the probability that a site is infected tends to 0 . 


\subsection{Bootstrap percolation}

The bootstrap percolation with the Duarte constraint is a discrete time dynamics of configurations on $\mathbb{Z}^{2}$; at each time $t \in \mathbb{N}$, any site of $\mathbb{Z}^{2}$ can be infected or healthy, and the states of the sites evolve according to the following rules: for any $t \in \mathbb{N}^{*}$,

- any site that is infected at time $t-1$ remains infected at time $t$;

- any site that is healthy at time $t-1$ becomes infected at time $t$ if and only if at time $t-1$, at least two sites among its top, left and bottom neighbors are infected.

This last condition is the Duarte constraint; a site can change state only if this constraint is satisfied (see Figure 7).

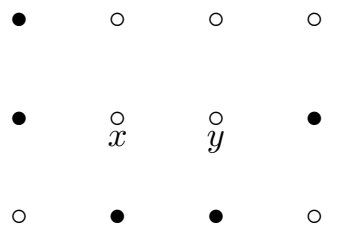

FigurE 7. Exemplification of the Duarte constraint. The $\bullet$ represent the infected sites and the o the healthy sites. Site $x$, which has two infected neighbors among the top, left and bottom ones, will be infected at the next step of the dynamics. Conversely, site $y$, which has only one infected neighbor among the top, left and bottom ones, will remain healthy at the next step of the dynamics.

One can notice that once a site is infected, it will remain so for ever: bootstrap percolation is monotone. Moreover, this dynamics is deterministic.

We may consider a random initial configuration in which the states of the sites are independent and the probability that any given site is infected is $q$, with $q \in[0,1]$ fixed; we call $\mu_{q}$ this law on configurations. The first question one can ask is: will the dynamics infect the origin with probability 1, or is there a positive probability that the origin remains healthy for ever? (We could ask the question for any other site, since the model is translation invariant.) The answer depends on $q$ :

Theorem 3.1 ( [34]). We have the following:

- if $q>0, \mu_{q}$ (the origin is infected by the dynamics $)=1$;

- if $q=0, \mu_{q}$ (the origin is infected by the dynamics $)=0$.

The property for $q=0$ is easy to see: if $q=0$, there are no infected sites in the initial configuration, hence the Duarte constraint is satisfied nowhere, and no site can become infected. Conversely, the result for $q>0$ requires a more complex proof, which was done by Roberto H. Schonmann in [34].

Theorem 3.1 implies that there is a phase transition at $q=0$. To understand how the dynamics goes from "almost sure infection of the origin" to "no infection at all of the origin" when $q$ becomes 0 , it is natural to consider the time at which the origin is infected,

$$
\tau_{B P}=\inf \{t \in \mathbb{N} \mid \text { the origin is infected at time } t\}
$$

and to study its behavior when $q$ tends to zero.

The following theorem was proven by Béla Bollobás, Hugo Duminil-Copin, Robert Morris and Paul Smith in [8] (a result on a closely related quantity called critical probability had been previously shown by Thomas Mountford in [31]).

Theorem 3.2 ( [8]). The median $T_{B P}$ of $\tau_{B P}$ satisfies $T_{B P}=\exp \left(\Theta\left(\frac{1}{q} \ln \left(\frac{1}{q}\right)^{2}\right)\right)$ when $q$ tends to $0^{1}$.

\footnotetext{
${ }^{1}$ If $f, g$ are two positive quantities depending on $q$, we write $f=\Theta(g)$ if $0<\liminf _{q \rightarrow 0} \frac{f}{g} \leq \limsup _{q \rightarrow 0} \frac{f}{g}<\infty$.
} 


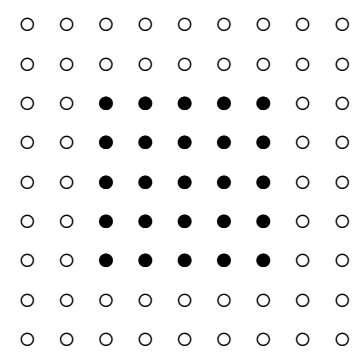

FigURE 8 . The $\bullet$ represent the infected sites and the $\circ$ the healthy sites. This isolated rectangle of infected sites does not allow to infect the neighboring sites.

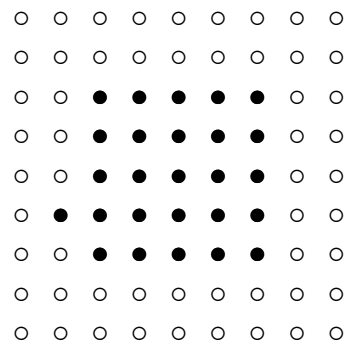

a)

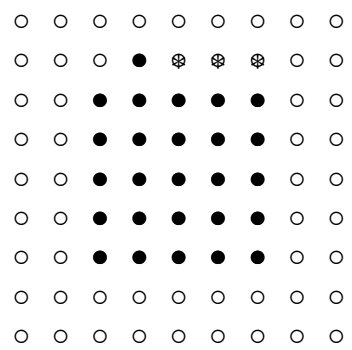

b)

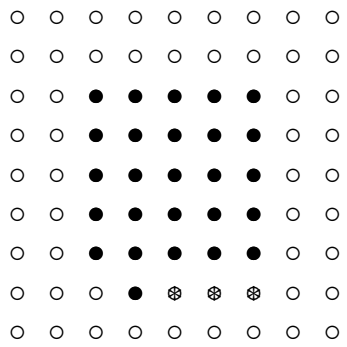

c)

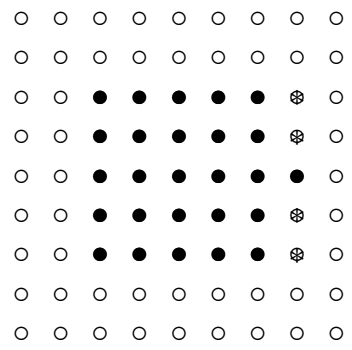

d)

FIgURE 9 . The $\bullet$ represent the initially infected sites, the $\otimes$ represent the initially healthy sites that can be infected by the dynamics, and the o represent the initially healthy sites that cannot be infected. We can see in a) that if we add to a rectangle of infected sites an infected site on its left side, we do not obtain any supplementary infection. In b) and c), we see that if we add the infected site on the top side (respectively the bottom side) of the rectangle, this allows to infect the part of the top side (respectively the bottom side) at the right of the added site. Finally, in d), we see that if we add the site on the right side of the rectangle, we can infect the entire right side.

We would like to replace the $\Theta$ with an exact constant, but such a result is not proven yet. An exact constant was shown for the critical probability by Béla Bollobás, Hugo Duminil-Copin, Robert Morris and Paul Smith in [9], but this constant cannot be transferred directly to $\tau_{B P}$.

Heuristics for Theorem 3.2. Firstly, we notice that since the probability that a site is infected in the initial configuration is $q$, which tends to zero, initially infected sites are scarce, so it is likely there are no initially infected sites around the origin. To reach the origin, the infection will have to start from a "seed", a place where there are initially infected sites, and to propagate to the origin.

Now, we can see on Figure 8 that an isolated rectangle of infected sites does not allow to propagate the infection; it cannot even infect the neighboring sites. Consequently, in order to propagate, the infection must encounter initially infected sites on its way.

Moreover, Figure 9 shows that when we add an infected site on a side of a rectangle of infected sites, the most effective propagation of infection is obtained when the added site is on the right side. Therefore, the optimal seed of infection must have a big right side so we have a large probability to find an initially infected site on it, and as few sites as possible because the more infected sites are required to form the seed, the less probable it is to find one; therefore the optimal seed is a column of infected sites.

To understand the legnth of the optimal column, we notice that the probability not to find an infected site on its right side is $(1-q)^{\mid \text {right side } \mid}=e^{-\Theta(q \mid \text { right side| })}$, so for this probability to be small, the right side must 
have length at least $\Theta\left(\frac{1}{q} \ln \left(\frac{1}{q}\right)\right)$. With such a length, there is a good probability to find an infected site on the right side of the column (then the mechanism of Figure $9 \mathrm{~d}$ ) allows to infect the entire right side, which is a new column), then on the right side of the newly infected column (which allows to infect this right side), then on the column on the right, etc. so infection can propagate over a certain length. As the optimal seed must contain as few sites as possible, it will be a column of $\Theta\left(\frac{1}{q} \ln \left(\frac{1}{q}\right)\right)$ initially infected sites. In addition, we see that the infection essentially propagates to the right.

We deduce that the infection that propagates to the origin comes from a column of $\Theta\left(\frac{1}{q} \ln \left(\frac{1}{q}\right)\right)$ initially infected sites at the left of the origin. Moreover, the probability that a given column of $\Theta\left(\frac{1}{q} \ln \left(\frac{1}{q}\right)\right)$ sites is entirely infected in the initial configuration is $q^{\Theta\left(\frac{1}{q} \ln \left(\frac{1}{q}\right)\right)}=\exp \left(\Theta\left(\frac{1}{q} \ln \left(\frac{1}{q}\right) \ln (q)\right)\right)=\exp \left(-\Theta\left(\frac{1}{q} \ln \left(\frac{1}{q}\right)^{2}\right)\right)$. Therefore, we can expect the initially infected column of height $\Theta\left(\frac{1}{q} \ln \left(\frac{1}{q}\right)\right)$ nearest to the origin to be at distance $\exp \left(\Theta\left(\frac{1}{q} \ln \left(\frac{1}{q}\right)^{2}\right)\right)$ from it; to cross this distance, the infection will take a time $\exp \left(\Theta\left(\frac{1}{q} \ln \left(\frac{1}{q}\right)^{2}\right)\right)$, hence the scaling of $\tau_{B P}$.

\subsection{Kinetically constrained model}

The kinetically constrained model with the Duarte constraint is also a dynamics of configurations on $\mathbb{Z}^{2}$ in which each site can be either infected or healthy, but this dynamics is stochastic and continuous-time.

To define the dynamics, one has to take independently for each site $x \in \mathbb{Z}^{2}$ a Poisson point process $\mathcal{P}_{x}$ on $\mathbb{R}^{+}$with parameter 1 . For each $t \in \mathcal{P}_{x}$, the site $x$ can receive an update at time $t$, which means having its state replaced by "infected" with probability $q$ and "healthy" with probability $1-q$ independently of everything else; $x$ receives an update at time $t$ if and only if the Duarte constraint is satisfied at time $t$, that is if and only if at least two sites among the top, left and bottom neighbors of $x$ are infected at time $t$ (see [11] for more details on the definition of kinetically constrained models).

The state of a site can only change via an update, therefore only when the Duarte constraint is satisfied, as in the bootstrap percolation Duarte model. However, one can notice that the occurrence of an update does not depend on the state of the site, neither does the new state of the site. In particular, an update may allow an infected site to become healthy, unlike in bootstrap percolation: the kinetically constrained model is not monotone.

As in the bootstrap percolation case, one can take an initial configuration of law $\mu_{q}$ (we denote by $\mathbb{P}_{q}$ the corresponding distribution of the process) and ask if there exists almost surely a time at which the origin is infected by the dynamics. It is not exactly the same question as the one we asked for bootstrap percolation, because when the origin is infected in the kinetically constrained model, it does not necessarily remain so for ever as it would in bootstrap percolation. Nevertheless, it is interesting to know if the origin is stuck forever in a healthy state or if its state has a chance to change. The answer is the same as for bootstrap percolation:

Theorem 3.3 ( [11]). We have the following:

- if $q>0, \mathbb{P}_{q}$ (the origin is infected by the dynamics $)=1$;

- if $q=0, \mathbb{P}_{q}$ (the origin is infected by the dynamics $)=0$.

The result for $q=0$ has the same origin as in bootstrap percolation: if $q=0$, there are no infected sites in the intial configuration, hence no update can occur, so no site can change state and get infected. The property for $q>0$ is harder to prove and can be obtained from the results of Nicoletta Cancrini, Fabio Martinelli, Cyril Roberto and Cristina Toninelli (see [11]).

Theorem 3.3 implies that there is a phase transition at $q=0$ in the kinetically constrained model. To understand it, it is again natural to consider the first time at which the origin is infected,

$$
\tau_{K C M}=\inf \left\{t \in \mathbb{R}^{+} \mid \text {the origin is infected at time } t\right\}
$$

and to study its behavior as $q$ tends to 0 .

There is an easy lower bound for $\tau_{K C M}$. Indeed, if a site gets infected in the kinetically constrained model, it received an update, hence the Duarte constraint was satisfied at this site, therefore it would have been infectable 
by the bootstrap percolation. Thanks to this, it can be proven that the infection in the kinetically constrained model cannot propagate much faster that in bootstrap percolation, more precisely (Lemma 4.3 of [28]) that when $q$ tends to zero ${ }^{2}$,

$$
\mathbb{E}_{q}\left(\tau_{K C M}\right)=\Omega\left(T_{B P}\right)=\exp \left(\Omega\left(\frac{1}{q} \ln \left(\frac{1}{q}\right)^{2}\right)\right) .
$$

However, this lower bound actually does not yield the true behavior of $\tau_{K C M}$, which is given by the following theorem:

Theorem $3.4([27,29]) \cdot \mathbb{E}_{q}\left(\tau_{K C M}\right)=\exp \left(\Theta\left(\frac{1}{q^{2}} \ln \left(\frac{1}{q}\right)^{4}\right)\right)$ when q tends to 0 .

The upper bound was shown by Fabio Martinelli, Robert Morris and Cristina Toninelli in [29], and the lower bound was proven by Laure Marêché, Fabio Martinelli and Cristina Toninelli in [27]. Theorem 3.4 implies that the first time at which the origin is infected diverges much faster in the kinetically constrained model than in bootstrap percolation: the two versions of the Duarte model have different behaviors.

Heuristics for Theorem 3.4. We already noticed that a site that gets infected by the kinetically constrained model would have been infectable by the bootstrap percolation, hence the infection that reaches the origin in the kinetically constrained model must come from a seed that allows to propagate infection to the origin in the bootstrap percolation dynamics: an initially infected column of height $\Theta\left(\frac{1}{q} \ln \left(\frac{1}{q}\right)\right)$ at the left of the origin.

Moreover, when the bootstrap percolation infects a site, its constraint is satisfied, hence it can receive an update in the kinetically constrained model, so it can get infected in the kinetically constrained model. Therefore, the mechanism by which the infection propagates to the origin in the bootstrap percolation model, by infecting successively the columns at the right of the initial column, may happen in the kinetically constrained model.

However, this will not happen in practice, because this would require the dynamics to go through a configuration where all columns between the initial column and the origin are infected, and since the probability that a site is infected when receiving an update is $q$ which tends to zero, it is very small, therefore the probability of infecting so many sites at the same time is really tiny. Before such an improbable event happens, we will have to wait for a long time. We deduce that what governs the scaling of $\tau_{K C M}$ is not so much that the infection has to cross a long distance from the initial infection seed to the origin, but the fact that before infecting the origin, the dynamics has to go through configurations that are very improbable because they contain too many infected sites. This phenomenon is called energy barrier. Therefore, to find the scaling of $\tau_{K C M}$, we have to find the optimal way for the dynamics to infect the origin, the one that uses as few infected sites as possible.

Since the smallest cluster of infected sites allowing to propagate infection, hence to act significantly on its environnement, is a column of height $\Theta\left(\frac{1}{q} \ln \left(\frac{1}{q}\right)\right)$, the optimal way to infect the origin will be having such columns move towards the origin. In what follows, we will write just "column" for "column of infected sites of height $\Theta\left(\frac{1}{q} \ln \left(\frac{1}{q}\right)\right)$ ".

We need to understand how these columns can move. We notice that such a column is long enough so we have a good probability to find an infected site on its right side, which allows to infect all its right side in the bootstrap percolation dynamics hence in the kinetically constrained model as in Figure 10. This implies that a column can create another column at its right. Moreover, if we have two successive columns in the configuration, the column at the right can disappear as evidenced by Figure 10. Therefore a column can appear or disappear when there is a column at its left. This columns dynamics resembles another kinetically constrained model, the East model, which is a dynamics of configurations on $\mathbb{Z}$ where a site can receive an update when the site at its left is infected (see [15]).

Moreover, in the East model, there is a combinatorial result from [12] claiming that before the infection can reach a distance $d$ from an initially infected site, the dynamics has to go through a configuration with at least $\log _{2} d$ infected sites at the same time, because when we allow only $n$ infected sites at the same time in the configuration, the infection can go to a distance roughly $2^{n}$ from the initial infected site. This can be seen by recursion. Indeed, if when we allow only $n$ infected sites at the same time, there exists a procedure $(\mathcal{P})$ that

\footnotetext{
${ }^{2}$ If $f, g$ are two positive quantities depending on $q$, we write $f=\Omega(g)$ if $\liminf _{q \rightarrow 0} \frac{f}{g}>0$.
} 

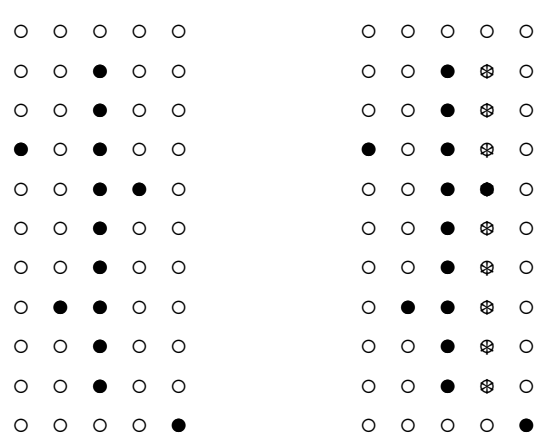

FiguRE 10. From the picture at the left with one infected column, where the $\bullet$ represent the infected sites and the $\circ$ the healthy sites, the dynamics can infect successively the sites $\otimes$, yielding the picture at the right with two infected columns. Moreover, from the picture at the right with two infected colums, the infected sites $\otimes$ can receive successive updates that make them healthy, which gives the picture at the left with a single infected column.

starts with only the origin of $\mathbb{Z}$ infected and infects $2^{n}$, then when we allow an $(n+1)$-th site, we can infect $2^{n}+1$ since its left neighbor is infected. We can then remove all the infected sites used to infect $2^{n}$ (apart from the origin) by reversing the procedure $(\mathcal{P})$, which yields a configuration with two infected sites, the origin and $2^{n}+1$. We now repeat $(\mathcal{P})$, but using $2^{n}+1$ as initially infected site instead of the origin. This allows to infect $\left(2^{n}+1\right)+2^{n}=2^{n+1}+1$, without using more than $n+1$ infected sites, so $n+1$ infected sites allow the infection to travel to a distance roughly $2^{n+1}$ from an initially infected site.

Therefore, as the columns in the Duarte model follow roughly the same dynamics as the infected sites in the East model, before a column can reach the origin to infect it, the dynamics has to go through a configuration with at least $\log _{2} d$ columns at the same time, where $d$ is the distance from the origin to the nearest initially infected column, which is expected to be $\exp \left(\Theta\left(\frac{1}{q} \ln \left(\frac{1}{q}\right)^{2}\right)\right)$. Consequently, before infecting the origin the dynamics will have to go through a configuration with at least $n$ columns at the same time, where $n=\log _{2}\left(\exp \left(\Theta\left(\frac{1}{q} \ln \left(\frac{1}{q}\right)^{2}\right)\right)\right)=\Theta\left(\frac{1}{q} \ln \left(\frac{1}{q}\right)^{2}\right)$. Since each column has probability $\exp \left(-\Theta\left(\frac{1}{q} \ln \left(\frac{1}{q}\right)^{2}\right)\right)$, such a configuration has probability $\left(e^{-\Theta\left(\frac{1}{q} \ln \left(\frac{1}{q}\right)^{2}\right)}\right)^{\Theta\left(\frac{1}{q} \ln \left(\frac{1}{q}\right)^{2}\right)}=\exp \left(-\Theta\left(\frac{1}{q^{2}} \ln \left(\frac{1}{q}\right)^{4}\right)\right)$. The time the dynamics will take before going through such an improbable configuration will be of order $\exp \left(\Theta\left(\frac{1}{q^{2}} \ln \left(\frac{1}{q}\right)^{4}\right)\right)$, which explains the scaling of $\tau_{K C M}$.

\section{Front PROPAGATION IN THE FA-1F MODEL}

\section{Aurelia Deshayes}

A key issue in the study of kinetically constrained models is to analyze the large time evolution when we start from a distribution different from the equilibrium Bernoulli measure $\mu_{q}$. Note that, due to the presence of the constraints, the KCM dynamics is not attractive ${ }^{3}$, so powerful tools like monotone coupling and censoring inequalities cannot be applied. Furthermore, convergence to equilibrium is not uniform on the initial condition since completely occupied configurations are blocked under the dynamics. Therefore the study of the large time dynamics out of equilibrium is particularly challenging.

In this section, we intend to present a progress in the understanding of kinetically constrained models (KCM), based on a joint work with Oriane Blondel and Cristina Toninelli [7]. We focus on the Fredrickson-Andersen one spin facilitated model (FA-1f) where the constraint requires at least one empty nearest neighbor. In particular, we consider FA-1f on $\mathbb{Z}$ starting from a configuration which has an empty site at the origin and is completely

\footnotetext{
${ }^{3} \mathrm{~A}$ dynamics is attractive if, the more empty sites the initial condition has, the more empty sites the process will have all the way long.
} 
occupied in the left half line and we study the evolution of the front, namely the position of the leftmost empty site. In the following, we will first present the FA-1f model and some of its properties related to convergence to equilibrium. Then, we will explain a coupling with an other interacting particle system, the contact process, which is not a KCM and satisfies attractivity. Finally, we will present the results on the front that we can deduce from the coupling.

\subsection{FA-1f model}

The FA-1f dynamics are described by a Markov process with the following generator: for any local function $f$ and any $\sigma \in\{0,1\}^{\mathbb{Z}}$,

$$
\mathcal{L} f(\sigma)=\sum_{x \in \mathbb{Z}} r(x, \sigma)\left(f\left(\sigma^{x}\right)-f(\sigma)\right)
$$

where the rate $r(x, \sigma)=c(x, \sigma)(q \sigma(x)+p(1-\sigma(x)))$ is the product between a constraint $c(x, \sigma)$ and a flip rate $q \sigma(x)+p(1-\sigma(x))$. The constraint $c(x, \sigma)=1-\sigma(x-1) \sigma(x+1)$, which requires at least one empty neighbor to allow the flip, does not depend on $\sigma(x)$ so that the FA-1f process is reversible w.r.t. $\mu_{q}:=\operatorname{Ber}(1-q)^{\otimes \mathbb{Z}}$. We denote by $L O_{0}$ the subspace of initial configurations $\sigma$ with a leftmost empty site at the origin, by $\sigma_{t}$ the configurations of the FA-1f model at time $t$ according to the previous dynamics, and by $X\left(\sigma_{t}\right)$ the front. As usual, $\mathbb{P}_{\sigma}$ denotes the law of the process started from the configuration $\sigma$. All those objects clearly depend on $q$, but to avoid burdensome reading we omit it from the notations.

First, it is easy to see that there is a maximal velocity of the front, that is, there exists a constant $\bar{v}$ such that, for every $\sigma \in L O_{0}$, every $c \geq \bar{v}$ and every $t$, we have $\mathbb{P}_{\sigma}\left(\left|X\left(\sigma_{t}\right)\right| \geq c t\right) \leq e^{-c t}$. This is an immediate consequence of the finite speed propagation of the information. To see that we use the graphical construction of the model: it is a space-time representation where the update times are marked by exponential clock rings (recall the beginning of Section 3.2). For an empty site to travel a distance $n$, it needs to cross at least $n$ independent clock rings, so the information cannot travel faster than linearly.

In [5] a convergence to equilibrium was proven when the starting distribution is such that the mean distance between nearest empty sites is uniformly bounded and the equilibrium vacancy density $q$ is larger than a threshold $1 / 2$. From their work we can easily deduce the following result.

Proposition 4.1. Let $q>1 / 2, t>0, K>0$ and $f$ be a bounded function with support contained in $[-K, K]$ such that $\mu_{q}(f)=0$. If $K \leq e^{t^{\alpha}}$ with $\alpha \in(0,1 / 2)$, there exists $c^{\prime}=c^{\prime}(\alpha, q)>0$ such that,

- if $\sigma$ has no interval of occupied sites of length $\sqrt{t}$ in $[-K-\bar{v} t, K+\bar{v} t]$ then $\left|\mathbb{E}_{\sigma}\left[f\left(\sigma_{t}\right)\right]\right| \leq \frac{1}{c^{\prime}}\|f\|_{\infty} e^{-c^{\prime} \sqrt{t}}$;

- if $\sigma$ has no interval of occupied sites of length $\sqrt{t}$ in $[-K, K]$ then $\left|\mathbb{E}_{\sigma}\left[f\left(\sigma_{t}^{[-K, K]}\right)\right]\right| \leq \frac{1}{c^{\prime}}\|f\|_{\infty} e^{-c^{\prime} \sqrt{t}}$, where $\left(\sigma_{t}^{[-K, K]}\right)_{t \geq 0}$ denotes the $F A-1 f$ process in $[-K, K]$ with empty boundary condition.

This result states that sufficiently many empty sites induce equilibrium. In the following, we will use this result and a coupling with threshold contact processes to understand the behavior of the front of a configuration. To be able to use the previous proposition, we will always consider the density of empty sites $q>1 / 2$.

\subsection{Coupling between FA-1f and contact process}

The threshold contact process presents similar dynamics to that of the FA-1f process but the update to occupy an empty site can occur without checking the constraint. In the contact process, the absorbing configuration with only occupied sites can be reached. Indeed, all the empty sites can disappear because there is no constraint the system has to satisfy in order to fill the last empty site. It is not the case for KCM.

We will denote by $\eta_{t}$ the configuration of the threshold contact process at time $t$ starting from $\eta$. This model is one of the simplest particle systems exhibiting a phase transition, that is, there exists $\bar{q} \in(0,1)$ such that if $q<\bar{q}$ then the probability to reach the absorbing state, starting from a configuration with a finite number of empty sites, is equal to 1 , and if $q>\bar{q}$ then the empty sites persist with positive probability. In the latter case, 
we say that the contact process survives, otherwise we say that the process dies. To ensure that the threshold contact process survives with positive probability, we will, from now on, suppose that $q>\bar{q}$.

Our idea is to couple FA-1f dynamics with a contact process in order to use the well-known behavior of the second process (ballistic motion of the front, large quantity of empty sites in the already visited zone) to study the first process.

Thanks to Harris graphical representation, starting from any initial configuration, we can construct both processes simultaneously using the same Bernoulli and Exponential variables. This construction, called basic coupling, ensures that every time a site updates from occupied to empty in the contact process, it is also emptied in the FA-1f process (but it is not the case for the update from empty to occupied because the FA-1f process requires the constraint). So if two initial configurations satisfy $\sigma \leq \eta$ (pointwise ${ }^{4}$ ), then a.s. for all time $t$, $\sigma_{t} \leq \eta_{t}$, so that coupled FA-1f and contact trajectories are obtained in such a way that FA-1f configurations contain more empty sites than contact process configurations.

We wish to exploit this comparison result between the FA-1f and contact processes to guarantee a sufficient number of empty sites for the FA-1f dynamics. To this purpose, since the contact process can die, we will need to build a restart argument first.

Lemma 4.2. Let $q>\bar{q}$. For any $\sigma \in L O_{0}$, there exist a process $\left(\sigma_{t}, \eta_{t}\right)_{t \geq 0}$ taking values in $\left(\{\text { empty, occupied }\}^{\mathbb{Z}}\right)^{2}$ and two random variables $T$ and $Y$ taking their values in $\mathbb{R}^{+}$and $\mathbb{Z}$, respectively, such that

(1) $\left(\sigma_{t}\right)_{t>0}$ is an $F A-1 f$ process starting from $\sigma \in L O_{0}$,

(2) $\forall t>0, \forall x \in \mathbb{Z}, \sigma_{t}(x) \leq \eta_{t}(x)$,

(3) $\left(\eta_{T+t}(Y+\cdot)\right)_{t>0}$ is a surviving threshold contact process starting from $\delta^{0}$, the configuration with a unique empty site at the origin.

Moreover, $T$ and $|Y|$ have exponentially decaying tail probabilities.

The idea of the proof is to couple a FA-1f process with a contact process and to restart the second one each time it vanishes. Eventually, the contact process will survive (since $q>\bar{q}$ ) and the space-time point $(Y, T$ ) corresponding to the origin of this surviving contact process is not very far from the origin (see Figure 11). The exponentially decaying tail probabilities come from the known behavior of the contact process: if the process dies, then it dies quickly (so the extinction times of the contact process tries, represented by the $U_{i}$ 's in Figure 11, are small) and its evolution is linear (so the growth of the contact process tries before extinction, represented by the $Z_{i}$ 's in Figure 11, are also small). Knowing that the FA-1f front has a maximal velocity, we can restart the process from a location close from the extinction point, that is the length between the origin of the previous contact process try and the origin of the new one (represented by the $X_{i}$ 's in Figure 11) are small.

Contrary to KCM, the contact process is attractive. This property is essential to prove its linear velocity and to ensure a good quantity of empty sites.

Thanks to this coupling we have been able to transport some properties from the threshold contact process to the FA-1f: prove that the front has a minimal velocity $\underline{v}>0$ and give lower bounds on the amount of empty sites.

\subsection{Results}

Combining the previous construction with Proposition 4.1, we obtain a result of relaxation far from the front. To do that we decorrelate the front trajectory from the interval in which we want to relax.

Theorem 4.3. Let $q>\bar{q}, \sigma \in L O_{0}, \alpha<1 / 2$ and $\delta>0$. There exists $c>0$ such that for any $M \leq e^{\delta t^{\alpha}}$, any $f$ with support in $[0, M], \mu(f)=0$ and $\|f\|_{\infty} \leq 1$, if $\sigma$ has no interval of occupied sites of length $\sqrt{t}$ in $[\underline{v} t, M+(4 \bar{v}-\underline{v}) t]$, then

where $\theta$ is the space shift operator.

$$
\left|\mathbb{E}_{\sigma}\left[f\left(\theta_{X\left(\sigma_{t}\right)+3 \bar{v} t} \sigma_{t}\right)\right]\right| \leq e^{-c \sqrt{t}}
$$

\footnotetext{
${ }^{4}$ For the order "empty< occupied".
} 


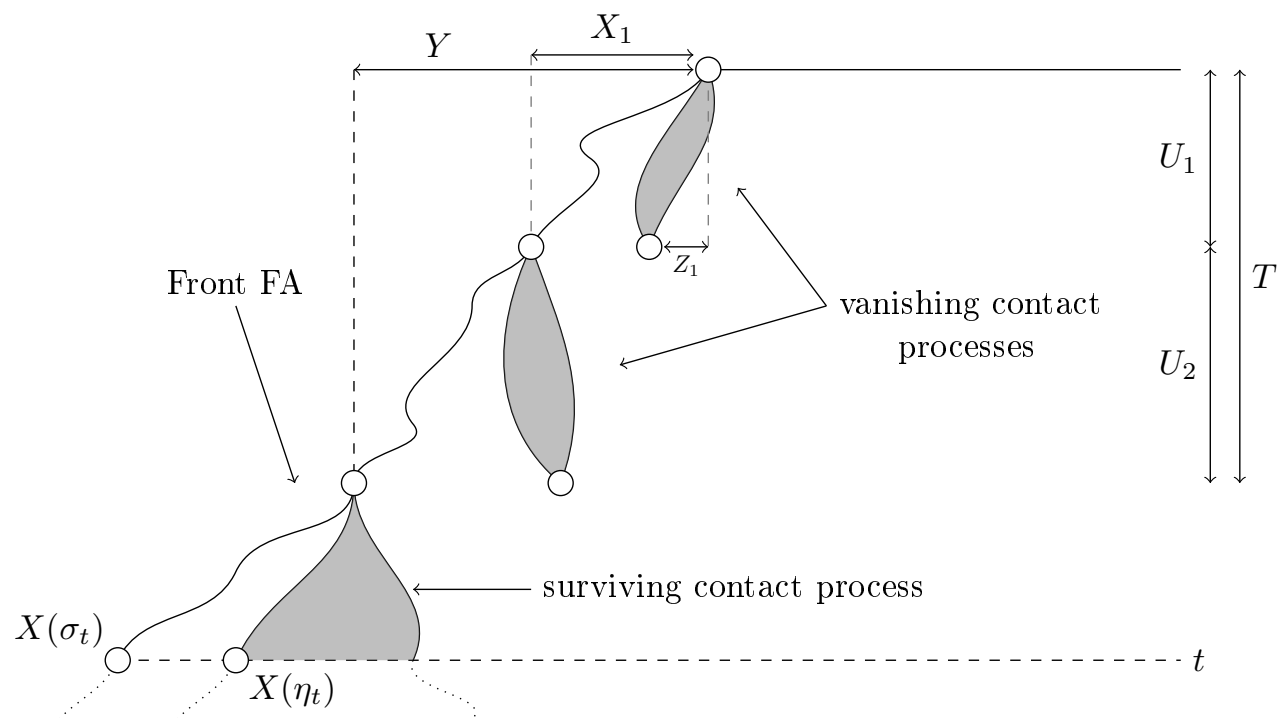

FigURE 11. Restart procedure, coupling between FA-1f and surviving contact process.
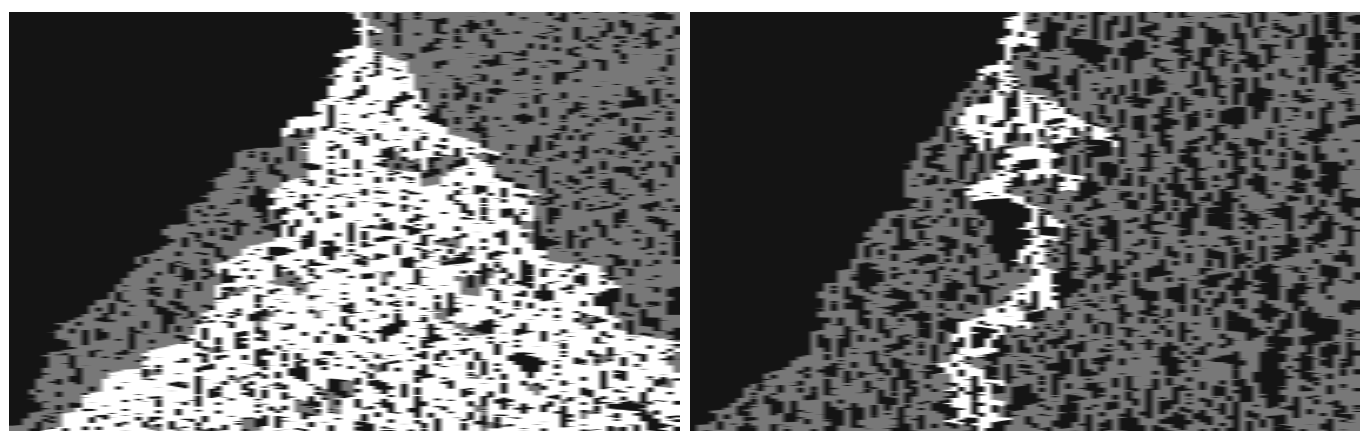

FiguRE 12. Simulation of FA-1f dynamics (with empty sites in gray) coupled with restart threshold contact processes (with empty sites in white). The first one is for $q>\bar{q}$ and the second one for $q<\bar{q}$.

Thanks to Theorem 4.3 we know that far from the front the configurations starting from $\sigma$ and $\sigma^{\prime}$ respectively will be close to a configuration sampled by the equilibrium measure, so they will be close to each other (for the total variation distance). Following the strategy of $[4,18]$, we constructed a coupling where we use this property and where we wait until the configurations also coincide near to the front to conclude the following ergodicity result.

Theorem 4.4. Let $q>\bar{q}$. The process seen from the front has a unique invariant measure $\nu$ and, starting from every $\sigma \in L O_{0}$, it converges in distribution to $\nu$ in the following sense: there exist $d^{*}>0$ and $c>0$ (independent of $\sigma$ ) such that for $t$ large enough

$$
\left\|\tilde{\mu}_{t}^{\sigma}-\nu\right\|_{\left[0, d^{*} t\right]} \leq e^{-c e^{(\log t)^{1 / 4}}}
$$

where $\tilde{\mu}_{t}^{\sigma}$ is the distribution of the configuration seen from the front at time $t$ starting from $\sigma$, i.e. $\theta_{X\left(\sigma_{t}\right)} \sigma_{t}$, and $\left\|\pi-\pi^{\prime}\right\|_{\Lambda}$ denotes the total variation distance between the marginals of $\pi$ and $\pi^{\prime}$ on $\Lambda$. 
This convergence result allows us to analyze the increments of the front and to deduce a law of large numbers. Finally, to study the fluctuations of the front, we generalize the strategy of [18] which is itself based on the result of Bolthausen [10] to establish a central limit theorem for non stationary random variables but satisfying a proper mixing condition.

Theorem 4.5. Let $q>\bar{q}$. There exist $s=s(q)$ and $v=v(q)$ such that for all $\sigma \in L O_{0}$

$$
\begin{array}{rlll}
\frac{X\left(\sigma_{t}\right)}{t} & \underset{t \rightarrow \infty}{\longrightarrow} & v \quad \mathbb{P}_{\sigma} \text {-almost surely } \\
\frac{X\left(\sigma_{t}\right)-v t}{\sqrt{t}} & \underset{t \rightarrow \infty}{\stackrel{d}{\longrightarrow}} \mathcal{N}\left(0, s^{2}\right) \quad \text { w.r.t. } \mathbb{P}_{\sigma}
\end{array}
$$

where $v=p \cdot \nu[\tilde{\sigma}(1)=0]-q$ is negative ( $\tilde{\sigma}$ denoting the random configuration with law $\nu$ ).

The motion of the front has also been analyzed in [4,18] for another one-dimensional KCM, the East model, for which the constraint requires the site at the right of $x$ to be empty: similar results, which imply a cutoff phenomenon have been established.

Unfortunately, these techniques cannot be easily generalized in dimension $d \geq 2$ and the shape theorems for kinetically constrained models are open questions in higher dimensions.

\section{REFERENCES}

[1] U. Basu and P. K. Mohanty: Active-absorbing-state phase transition beyond directed percolation: a class of exactly solvable models, Physical Review E, 79, 041143 (2009).

[2] I. Benjamini, N. Berger, C. Hoffman and E. Mossel: Mixing times of the biased card shuffling and the asymmetric exclusion process, Transactions of the American Mathematical Society, 357(8), 3013-3029 (electronic) (2005).

[3] L. Bertini and G. Giacomin: Stochastic Burgers and KPZ equations from particle systems, Commun. Math. Phys. 183(3), pages 571-607 (1997).

[4] O. Blondel: Front progression in the East model, Stochastic Processes and their Applications, 123(9), 3430-3465 (2013).

[5] O. Blondel, N. Cancrini, F. Martinelli, C. Roberto, and C. Toninelli: Fredrickson-Andersen one spin facilitated model out of equilibrium, Markov Processes and Related Fields, 19(3), 383-406 (2013).

[6] O. Blondel, C. Erignoux, M. Sasada and M. Simon: Hydrodynamic limit for a facilitated exclusion process, Annales de l'Institut Henri Poincaré (B) Probabilités et Statistiques, 56(1) (2020)

[7] O. Blondel, A. Deshayes and C. Toninelli: Front evolution of the Fredrickson-Andersen one spin facilitated model, Electronic Journal of Probability, 24, 1-32 (2019).

[8] B. Bollobás, H. Duminil-Copin, R. Morris and P. Smith: Universality of two-dimensional critical cellular automata, Proceedings of the London Mathematical Society, to appear.

[9] B. Bollobás, H. Duminil-Copin, R. Morris and P. Smith: The sharp threshold for the Duarte model, Annals of Probability, 45, 4222-4272 (2017).

[10] E. Bolthausen: On the central limit theorem for stationary mixing random fields, Annals of Probability, 10(4), 1047-1050 (1982).

[11] N. Cancrini, F. Martinelli, C. Roberto and C. Toninelli: Kinetically constrained spin models, Probability Theory and Related Fields, 140(3-4), 459-504 (2008).

[12] F. Chung, P. Diaconis and R. Graham: Combinatorics for the East model, Advances in Applied Mathematics, 27, 192-206 (2001).

[13] A. De Masi, E. Presutti, and E. Scacciatelli: The weakly asymmetric simple exclusion process, Annales de l'Institut Henri Poincaré, Probabilités et Statistiques, 25(1):1-38 (1989).

[14] A. De Masi, E. Presutti, D. Tsagkarogiannis, and M.E. Vares: Current reservoirs in the simple exclusion process, Journal of Statistical Physics, 144, 1151-1170 (2011).

[15] A. Faggionato, F. Martinelli, C. Roberto and C. Toninelli: The East model: recent results and new progresses, Markov Processes and Related Fields, 19(3), 407-452, (2013).

[16] T. Funaki: Free boundary problem from stochastic lattice gas model, Annales de l'Institut Henri Poincaré, Probabilités et Statistiques, 35(5), 573-603 (1999).

[17] A. Gabel, P. L. Krapivsky and S. Redner: Facilitated asymmetric exclusion, Physical Review Letters, 105, 210603 (2010).

[18] S. Ganguly, E. Lubetzky, and F. Martinelli: Cutoff for the East process, Communications in Mathematical Physics, 335(3), $1287-1322(2015)$. 
[19] J. Gärtner: Convergence towards Burgers' equation and propagation of chaos for weakly asymmetric exclusion processes, Stochastic Processes and their Applications, 27(2), 233-260 (1988).

[20] C. Kipnis and C. Landim: Scaling limits of interacting particle systems, Grundlehren der Mathematischen Wissenschaften [Fundamental Principles of Mathematical Sciences], vol. 320, Springer-Verlag, Berlin, 1999.

[21] C. Labbé and H. Lacoin: Cutoff phenomenon for the asymmetric simple exclusion process and the biased card shuffling, Annals of Probability, Annals of Probability, 47(3) (2019).

[22] C. Labbé and H. Lacoin: Mixing time and cutoff for the weakly asymmetric simple exclusion process, ArXiv preprint: 1805.12213 (2018).

[23] H. Lacoin: Mixing time and cutoff for the adjacent transposition shuffle and the simple exclusion, Annals of Probability, 44(2), $1426-1487$ (2016).

[24] D. A. Levin and Y. Peres: Mixing of the exclusion process with small bias, Journal of Statistical Physics, 165(6), 1036-1050 (2016).

[25] D. A. Levin, Y. Peres and E. L. Wilmer: Markov chains and mixing times. American Mathematical Society, Providence, RI, 2017. Second edition of [ MR2466937], With a chapter on "Coupling from the past" by James G. Propp and David B. Wilson.

[26] T. M. Liggett: Stochastic Interacting Systems: Contact, Voter and Exclusion Processes, Grundlehren der Mathematischen Wissenschaften vol 324, Berlin: Springer, 1999.

[27] L. Marêché, F. Martinelli and C. Toninelli: Exact asymptotics for Duarte and supercritical rooted kinetically constrained models, Annals of Probability, 48(1) (2020).

[28] F. Martinelli and C. Toninelli: Towards a universality picture for the relaxation to equilibrium of kinetically constrained models, Annals of Probability, 47(1), 324-361 (2019).

[29] F. Martinelli, R. Morris and C. Toninelli: Universality results for kinetically constrained spin models in two dimensions, Communications in Mathematical Physics, 369(2), 761-809 (2019).

[30] H. Meirmanov: The Stefan Problem, (Translated from the Russian). De Gruyter Expositions in Mathematics, Vol. 3, Walter de Gruyter \& Co., Berlin, 1992.

[31] T. Mountford: Critical length for semi-oriented bootstrap percolation, Stochastic Processes and their Applications, 56(2), 185-205 (1995).

[32] F. Ritort and P. Sollich: Glassy dynamics of kinetically constrained models, Advances in Physics, 52(4), 219-342 (2003).

[33] M. Rossi, R. Pastor-Satorras, and A. Vespignani: Universality class of absorbing phase transitions with a conserved field, Physical Review Letters, 85, 1803 (2000).

[34] R. H. Schonmann: Critical points of two-dimensional bootstrap percolation-like cellular automata, Journal of Statistical Physics, $\mathbf{5 8}(5 / 6), 1239-1244(1990)$.

[35] J. Stefan: Über die Theorie der Eisbildung, insbesondere über die Eisbildung im Polarmeere, Annalen der Physik und Chemie, 42, 269-286 (1891).

[36] D. B. Wilson: Mixing times of lozenge tiling and card shuffling Markov chains, Annals of Applied Probability, 14(1), 274-325 (2004). 